\title{
Cognitive and Functional Abilities in an Older Adult Veteran Before and After Contracting COVID-19
}

\author{
Ronald Okolichany $^{\mathrm{a}, \mathrm{d}, *}$, Prasad R. Padala ${ }^{\mathrm{a}, \mathrm{b}, \mathrm{c}}$ and Scott Mooney ${ }^{\mathrm{d}}$ \\ ${ }^{a}$ Geriatric Research Education and Clinical Center (GRECC), Central Arkansas Veterans Healthcare System, \\ North Little Rock, AR, USA \\ ${ }^{\mathrm{b}}$ Department of Psychiatry, University of Arkansas for Medical Sciences, Little Rock, AR, USA \\ ${ }^{\mathrm{c}}$ Baptist Health/UAMS Graduate Medical Education, North Little Rock, AR, USA \\ ${ }^{\mathrm{d}}$ Neuropsychology Service, Central Arkansas Veterans Healthcare System, North Little Rock, AR, USA
}

Received 26 October2021

Accepted 12 February 2022

Pre-press 5 March 2022

Published 25 March 2022

\begin{abstract}
.
Background: A 76-year-old male Veteran with a historical diagnosis of mild cognitive impairment was assessed at baseline and follow-up as part of two separate, ongoing studies. He was diagnosed with COVID-19 during the interim.

Objective: To report potential effects on cognitive and functional abilities measured before and after contracting COVID-19. Methods: The patient was administered a series of cognitive tests and self-report procedures assessing cognitive, functional, and neuropsychiatric status.

Results: Overall, no discernable pattern of cognitive changes between pre-COVID and post-COVID assessments were noted. Only mild increases in agitation, depression, and irritability were noted on a self-report measure. However, this particular subject has relatively ideal psychosocial circumstances in comparison to the typical older adult Veteran male. It is hypothesized that improved psychosocial conditions will result in less negative cognitive and functional outcomes for older adults diagnosed with COVID-19.
\end{abstract}

Conclusion: High levels of resilience, social support, and exercise, coupled with lower levels of perceived stress and loneliness may serve as protective factors against cognitive and functional decline in older adults who contract COVID-19.

Keywords: Cognition, COVID-19, geriatrics, neuropsychology, Veterans

\footnotetext{
*Correspondence to: Ronald Okolichany, Geriatric Research Education and Clinical Center (GRECC), Central Arkansas Veterans Healthcare System, 2200 Fort Roots Dr., North Little Rock, AR 72114, USA. Tel.: +1 501257 2537; Fax: +1 501257 2501; E-mail: Ronald.Okolichany1@va.gov.
}

\section{INTRODUCTION}

The COVID-19 pandemic began in December 2019 in Wuhan, China and continues through the time of this study. It has since been declared a global public health crisis and has overwhelmed healthcare 
systems worldwide. On October 25, 2021, there were 243,260,214 confirmed global cases with 4,941,039 recorded deaths according to the World Health Organization [1]. The economic, sociocultural, and psychological impact has been immense. Elevated levels of anxiety, depression, posttraumatic stress disorder, psychological distress, and stress related to the COVID-19 pandemic have been reported in several countries [2]. The mental health impact seems clear and far reaching. What is less clear is the extent of the impact of COVID-19 infection on cognition.

Information regarding cognitive impairments following symptomatic contraction of COVID-19 and subsequent recovery has been mixed. Cognitive impairment has been hypothesized to be a deleterious effect resulting from the immunoinflammatory process in other viral infections [3]. The COVID19 virus may also enter the central nervous system (CNS) through the nasal mucosa and olfactory fibers, potentially infecting neurons [4]. The neuropathological mechanisms by which COVID-19 may lead to neuronal injury include hypoxia (e.g., secondary to pneumonia and concomitant peripheral vasodilation and hypercarbia) and immune mediated injury (e.g., increased levels of inflammatory cytokines and vascular leakage secondary to Interleukins 6 release). This may lead to encephalopathy and delirium with other complications such as cerebrovascular accident, Guillian-Barré syndrome, acute transverse myelitis, and acute encephalitis [5]. Delirium is among the most common sources of cognitive impairment in the acute stages of the disease. Individuals with COVID-19 are at increased risk for delirium due to factors such as direct CNS invasion, CNS inflammatory mediators, organ failure, sedative administration, prolonged mechanical ventilation, immobilization, and social isolation [6]. Cognitive sequelae have also been reported following recovery from COVID19. Zhou et al. [3] found evidence of impairments in sustained attention as measured by a continuous performance task in a population of recovered COVID-19 patients. Impaired cognitive performance has also been measured several months following hospital discharge, including increased fatiguability, decreased processing speed, and deficits in concentration and memory [7]. A systematic review of 33 studies examining recovered or recovering COVID19 patients found elevated rates of depression, anxiety, fatigue, sleep disruption, and posttraumatic stress. Deficits in attention, executive functioning, and memory were most consistently reported across studies [8].
While several articles have been published regarding possible cognitive impact following COVID-19 exposure, none, to the knowledge of this author, have had objective cognitive data before and after exposure available for comparison. The aim of this paper is to present a case study in which cognitive data was collected before and after COVID-19 infection. Of note, this data was derived from two independent studies. It was an adventitious occurrence that a participant contracted COVID-19 independently between cognitive evaluations. The measurements obtained are idiosyncratic to those studies and do not provide an ideal assessment of the full range of cognitive domains. However, we argue that there are still valuable inferences to be extrapolated regarding the cognitive impact of COVID-19. Further, the present case possesses relatively pristine psychosocial circumstances toward which outcomes of older adults with greater psychosocial challenges may be compared. It has been demonstrated in several studies that psychosocial factors such as self-efficacy [9], positive affect [10], social engagement [11, 12], and social support [13] have a protective effect on cognition later in life.

\section{CASE DESCRIPTION}

Mr. A is a 76-year-old, married, Caucasian, male, Veteran with 15 years of formal education living at home with his spouse in a rural area with low access to care (i.e., 79 miles from VA medical center). He is currently retired from a position in law enforcement/criminal justice. He was recruited to participate in two separate research studies from which the present data was obtained. Informed consents were obtained from the patient for the two studies (detailed below) from which the data were derived. He provided written consent for this case report as well.

The Veteran contracted COVID-19 in March 2020. Additional past medical history is also positive for mild cognitive disorder, depression, obstructive sleep apnea, hyperlipidemia, hypertension, arteriosclerosis of coronary artery bypass graft, and gastroesophageal reflux disease. His medication list included amlodipine, carboxymethylcellulose, clopidogel, escitalopram, esomeprazole, fluticasone, hydrochlorothiazide, isosorbide mononitrate, metoprolol tartrate, ranolazine, rosuvastatin, aspirin, and loratadine. A head CT conducted five years prior to the present study revealed mild cerebral volume loss and periventricular white matter changes. Substance 
use history is reportedly positive for remote tobacco and cannabis use (last use was 34 years ago). He was diagnosed with mild cognitive impairment, amnestic type following a brief neuropsychological evaluation conducted three years prior to the present study.

\section{Measures}

The New York University Paragraph Recall Test (NYU Paragraphs) [14], Mini-Mental Status Exam (MMSE) [15], and The Conners Continuous Performance Test -Third Edition [16] were obtained on two occasions as part of a study investigating the cognitive effects of transdermal nicotine therapy on MCI. All other data was obtained from medical chart review and a study investigating the effects of COVID-19 restrictions on older adults with cognitive impairment. The obtained measures along with a brief description are as follows: The NYU Paragraphs consists of orally presented contextual verbal information with immediate and delayed memory components. It is useful for predicting whether cognitive decline will progress to dementia [14, 17]. The MMSE is a brief cognitive screening measure encompassing domains such as spatial and temporal orientation, confrontation naming, visuospatial skills, repetition, executive functioning, registration/learning, delayed memory, and serial subtraction. The CPT- 3 is a computerized vigilance test which assesses attentional capacity. The test takes 14 minutes to complete, during which time the examinee must maintain a constant state of vigilance. The test provides a number of measures which assess varying aspects of attention and impulsivity. It is resistant to practice effects [16].

The following assessments and self-report measures were obtained as part of a study examining the effects of COVID-19 pandemic on neuropsychiatric functioning of individuals with dementia: Telephone Montreal Cognitive Assessment (T-MoCA) [18] a brief, telephone-administered cognitive screening measure encompassing simple attention, vigilance, spatial and temporal orientation, repetition, phonemic fluency, registration/learning, delayed memory, serial subtraction, and abstraction; expanded COWAT (i.e., CFLJW) [19] - a measure of effort and phonemic verbal fluency; Animal Naming - a task of semantic verbal fluency; Brief Resilient Coping Scale (BRCS) [20] - a measure of coping ability and attitudes regarding adverse experiences; International Physical Activity Questionnaire (IPAQ) [21] - a measure examining exercise frequency and intensity; Three-Item Loneliness Scale (LS-3) [22] - a measure of subjective loneliness and feelings of isolation; Lubben Social Network Scale (LSNS) [23] - a measure of social activity and social support; Functional Activities Questionnaire (FAQ) [24] - a measure of functional ability regarding instrumental activities of daily living; Neuropsychiatric Inventory Questionnaire (NPI-Q) [25] - a measure of behavioral and psychiatric disturbance and associated severity modified to assess change during the COVID-19 pandemic; and Perceived Stress Scale 4 (PSS-4) [26] an assessment of subjective life stress and locus of control.

\section{Procedures}

CPT-3, MMSE, and NYU paragraphs were first administered in January of 2020 as part of a study examining the effects of nicotine patch on MCI. The subject tested positive for COVID-19 in March 2020. Follow up cognitive testing encompassing CPT-3, NYU Paragraphs, and MMSE was conducted in July 2020. See Table 1 for comparison.

Following his participation in the nicotine study, he enrolled in a study examining the effects of COVID-19 on individuals with dementia. The measures illustrated in Table 2 were obtained in March 2021.

MMSE Total Scores were intact at both initial assessment (January 2020) and follow up (July 2020). Attentional measures were also intact at follow up and scores did not differ significantly based on reliable change index [27]. Delayed recall on NYU Paragraphs was above $100 \%$ for initially encoded information for both evaluations. At the time of third measurement, the subject demonstrated credible task engagement with intact verbal fluency and T-MoCA total score, high levels of resilience, exercise, social engagement, and low levels of loneliness, stress, and functional impairment. Mild increases in neuropsychiatric symptoms including agitation, depression, appetite, and irritability were noted on the NPI-Q.

\section{DISCUSSION}

Other respiratory infections which have resulted in global health crises have been associated with adverse neuropsychiatric changes. For instance, severe acute respiratory syndrome (SARS) which originated in 2003 was associated with reported memory impairments, poor concentration, anxiety, 
Table 1

Procedures administered at baseline (January 2020) and follow-up (July 2020)

\begin{tabular}{lcc}
\hline Domain & $\begin{array}{c}\text { Pre-COVID } \\
\text { (January 2020) }\end{array}$ & $\begin{array}{c}\text { Post-COVID } \\
\text { (July 2020) }\end{array}$ \\
\hline Attention & & \\
$\quad$ CPT Omissions & 42.97 & 42.97 \\
CPT Commissions & 53.66 & 44.95 \\
Hit RT Std. Error & 47.32 & 47.41 \\
MMSE Attn/Calc & $5 / 5$ & $5 / 5$ \\
Orientation & & \\
$\quad$ MMSE Orientation & $9 / 10$ & $8 / 10$ \\
Memory & $7 / 19(36.84 \%)$ & $9 / 21(42.86 \%)$ \\
$\quad$ NYU Paragraphs - Immediate & $9 / 19(47.37 \%)$ & $11 / 21(52.38 \%)$ \\
$\quad$ NYU Paragraphs - Delayed & $3 / 3$ & $3 / 3$ \\
MMSE Registration & $3 / 3$ & $2 / 3$ \\
$\quad$ MMSE Recall & & $27 / 30$ \\
Global & $28 / 30$ & \\
$\quad$ MMSE Total Score &
\end{tabular}

MMSE Total Scores were intact at both initial assessment (January 2020) and follow up (July 2020). Attentional measures were also intact at follow up and scores did not differ significantly based on reliable change index. Delayed recall on NYU Paragraphs was above $100 \%$ for initially encoded information for both evaluations.

Table 2

Further cognitive and neuropsychiatric measures obtained in March 2021

\begin{tabular}{lc}
\hline Domain & Score \\
\hline Effort & \\
$\quad$ CFLJW & $\mathrm{Z}=-0.19$ (Valid) \\
Verbal Fluency & $\mathrm{Z}=-0.43$ \\
$\quad$ CFL & $\mathrm{T}=44$ \\
$\quad$ Animals & \\
Global & $20 / 22$ \\
$\quad$ T-MoCA Total Score & \\
& \\
Self-Report Measure & $20 / 20$ \\
BRCS & $2 \mathrm{~h}$ of walking/day, 7 days/week \\
IPAQ & $3 / 9$ \\
LS-3 & $30 / 30$ \\
LSNS & $0 / 16$ \\
PSS-4 & $0 / 30$ \\
FAQ & $4 / 12$ \\
NPI-Q Total Symptoms Increased &
\end{tabular}

At the time of third measurement, the subject demonstrated credible task engagement with intact verbal fluency and T-MoCA total score, high levels of resilience, exercise, social engagement, and low levels of loneliness, stress, and functional impairment. Mild increases in neuropsychiatric symptoms including agitation, depression, appetite, and irritability were noted on the NPI-Q.

depression, insomnia, and tension [28]. Information regarding the neuropsychiatric impact of the COVID19 pandemic is sparse but increasingly available. The short-term effects of COVID-19 appeared to increase depression rates among older adults [29], likely associated with lockdown, quarantine, and social distancing measures implemented to reduce the spread of the virus which have disrupted the social landscape. However, there is evidence to sug- gest that the short-term impact of the pandemic was not as severe on older adults' wellbeing as the longterm effects of protracted pandemic restrictions [30]. Resilience, or the ability to recover from adverse experiences, appears to be emerging as a major mediator for mental and physical health outcomes in older adults during the COVID-19 pandemic [31]. Older adults with higher levels of resilience tend to age more successfully, experience lower levels of depression, and demonstrate increased longevity [32]. Subjective levels of cognitive decline in older adults during the COVID-19 pandemic may only be evident in a minority of older adults (8\%), with depression acting as a major risk factor in those who report declines [30]. However, in individuals with preexisting dementia and mild cognitive impairment, lockdown measures appear to increase the rate of decline [33].

Whether following direct COVID-19 infection or as indirect effects of lockdown measures, a number of potential neuropsychiatric and functional sequelae attributable to the COVID-19 pandemic have been proposed in the burgeoning literature. Coronavirus infection may result in neuronal injury through direct invasion of neurons or through secondary mechanisms such as hypoxia, immune-mediated injury, organ failure, and prolonged mechanical ventilation, leading to cognitive sequelae such as delirium and impaired sustained attention. Evidence for deficits in processing speed, concentration, and memory have been found months after hospital discharge. Rate of cognitive decline appears to accelerate in individuals with preexisting dementia and MCI as a result of lock- 
down measures, with depression emerging as at least one risk factor for subjective cognitive decline and resilience as a protective factor. It is currently unclear what factors may influence cognitive and functional abilities following COVID-19 infection. Studies published on cognitive sequelae following COVID-19 infection have thus far lacked baseline pre-infection cognitive testing for comparison. Thus, it is unclear whether those results represent true declines in cognition or characteristics of the population sampled. In this study, a case is presented in which objective cognitive data was collected before and after COVID-19 infection.

Overall, no discernable pattern of cognitive changes between pre-COVID and post-COVID assessments were found in the present study. Mild increases in neuropsychiatric symptoms including agitation, depression, appetite, and irritability were reported. However, it is possible that these neuropsychiatric symptoms are better attributed to lockdown measures rather than infection sequelae given that the majority of COVID-19 survivors make a full recovery. Furthermore, the current patient did not report delirium which has been the most consistent cognitive presentation of COVID-19 infection, further supporting the hypothesis that the neuropsychiatric symptoms are better attributed to lockdown measures. This particular case did not demonstrate the attention, concentration, and memory deficits noted in previous research. Granted, the potential effects of the nicotine study from which his cognitive data were obtained are unclear. It is known from preclinical studies on neuronal nicotinic receptors that attention, learning, and memory function may improve with nicotine administration [34]. Thus, it may potentially account for preservation cognitive abilities if he was in the experimental condition. Of note, this particular subject has relatively ideal psychosocial circumstances in comparison to the typical older adult Veteran male. This subject possessed a range of protective factors noted on self-report measures which may provide guidance for targeted interventions designed to mitigate the impact of COVID-19 infection and lockdown measures on cognitive functioning. It is hypothesized that improved psychosocial conditions will result in less negative cognitive and functional outcomes for older adults diagnosed with COVID-19. High levels of resilience, social support, and exercise, coupled with lower levels of perceived stress and loneliness, may serve as protective factors against cognitive and functional decline in older adults who contract COVID-19.

\section{ACKNOWLEDGMENTS}

The authors have no acknowledgments to report.

\section{FUNDING}

The authors have no funding to report.

\section{CONFLICT OF INTEREST}

The authors have no conflict of interest to report.

\section{REFERENCES}

[1] World Health Organization (2021) WHO Coronavirus (COVID-19) Dashboard. World Health Organization. https://covid19.who.int, Last updated October 25, 2021, Accessed on October 25, 2021.

[2] Xiong J, Lipsitz O, Nasri F, Lui LMW, Gill H, Phan L, Chen-Li D, Iacobucci M, Ho R, Majeed A, McIntyre RS (2020) Impact of COVID-19 pandemic on mental health in the general population: A systematic review. J Affect Disord 277, 55-64.

[3] Zhou H, Lu S, Chen J, Wei N, Wang D, Lyu H, Shi C, Hu $S$ (2020) The landscape of cognitive function in recovered COVID-19 patients. J Psychiatr Res 129, 98-102.

[4] Miners S, Kehoe PG, Love S (2020) Cognitive impact of COVID-19: Looking beyond the short term. Alzheimers Res Ther 12, 170 .

[5] Ahmad I, Rathore FA (2020) Neurological manifestations and complications of COVID-19: A literature review. J Clin Neurosci 77, 8-12.

[6] Kotfis K, Williams Roberson S, Wilson JE, Dabrowski W, Pun BT, Ely EW (2020) COVID-19: ICU delirium management during SARS-CoV-2 pandemic. Crit Care 24, 176.

[7] Ferrucci R, Dini M, Groppo E, Rosci C, Reitano MR, Bai F, Poletti B, Brugnera A, Silani V, D'Arminio Monforte A, Priori A (2021) Long-lasting cognitive abnormalities after COVID-19. Brain Sci 11, 235.

[8] Vanderlind WM, Rabinovitz BB, Miao IY, Oberlin LE, Bueno-Castellano C, Fridman C, Jaywant A, Kanellopoulos D (2021) A systematic review of neuropsychological and psychiatric sequalae of COVID-19: Implications for treatment. Curr Opin Psychiatry 34, 420-433.

[9] Windsor TD, Anstey KJ (2008) A longitudinal investigation of perceived control and cognitive performance in young, midlife and older adults. Aging Neuropsychol Cogn 15, 744-763.

[10] Paterson TSE, Yeung SE, Thornton WL (2015) Positive affect predicts everyday problem-solving ability in older adults. Aging Mental Health 20, 871-879.

[11] Kruger KR, Wilson RS, Kamenetsky JM, Barnes LL (2009) Social engagement and cognitive function in old age. Exp Aging Res 35, 45-60.

[12] Lövdén M, Ghisletta P, Lindenberger U (2005) Social participation attenuates decline in perceptual speed in old and very old age. Psychol Aging 20, 423-434.

[13] Noguchi T, Nojima I, Inoue-Hirakawa T, Sugiura H (2019) The association between social support sources and cognitive function among community-dwelling older adults: 
A one-year prospective study. Int J Environ Res Public Health 16, 4228.

[14] Kluger A, Ferris SH, Golomb J, Mittelman MS, Reisberg B (1999) Neuropsychological prediction of decline to dementia in nondemented elderly. J Geriatr Psychiatry Neuro 12, 168-179.

[15] Folstein MF, Folstein SE, McHugh PR (1975) "Minimental state". A practical method for grading the cognitive state of patients for the clinician. J Psychiatr Res 12, 189-198.

[16] Conners KC (2014) Conners Continuous Performance Test, 3rd Edition Technical Manual. Multi-Health Systems, Inc. Tonawanda, NY.

[17] Fleisher AS, Sowell BB, Taylor C, Gamst AC, Petersen RC, Thal LJ (2007) Clinical predictors of progression to Alzheimer disease in amnestic mild cognitive impairment. Neurology 68, 1588-1595.

[18] Pendlebury ST, Welch SJ, Cuthbertson FC, Mariz J, Mehta Z, Rothwell PM (2013) Telephone assessment of cognition after transient ischemic attack and stroke: Modified telephone interview of cognitive status and telephone Montreal Cognitive Assessment versus face-to-face Montreal Cognitive Assessment and neuropsychological battery. Stroke 44, 227-229.

[19] Silverberg ND, Hanks RA, Buchanan L, Fichtenberg N, Millis SR (2008) Detecting response bias with performance patterns on an expanded version of the Controlled Oral Word Association Test. Clin Neuropsychol 22, 140-157.

[20] Sinclair VG, Wallston KA (2004) The development and psychometric evaluation of the Brief Resilient Coping Scale. Assessment 11, 94-101.

[21] Craig CL, Marshall AL, Sjostrom M, Bauman AE, Booth ML, Ainsworth BE, Pratt M, Ekelund U, Yngve A, Sallis J, Oja P (2003) International physical activity questionnaire: 12 -country reliability and validity. Med Sci Sports Exerc 35, 1381-1395.

[22] Hughes ME, Waite LJ, Hawkley LC, Cacioppo JT (2004) A short scale for measuring loneliness in large surveys: Results from two population-based studies. Res Aging 26, 655-672.

[23] Lubben J, Blozik E, Gillmann G, Iliffe S, von Renteln Kruse W, Beck JC, Stuck AE (2006) Performance of an abbreviated version of the Lubben Social Network Scale among three European community-dwelling older adult populations. Gerontologist 46, 503-513.
[24] Pfeffer RI, Kurosaki TT, Harrah CH Jr., Chance JM, Filos S (1982) Measurement of functional activities in older adults in the community. J Gerontol 37, 323-329.

[25] Cummings JL, Mega M, Gray K, Rosenberg-Thompson S, Carusi DA, Gornbein J (1994) The Neuropsychiatric Inventory: Comprehensive assessment of psychopathology in dementia. Neurology 44, 2308-2314.

[26] Cohen S, Kamarck T, Mermelstein R (1983) A global measure of perceived stress. J Health Soc Behav 24, 385-396.

[27] Jacobson NS, Truax P (1991) Clinical significance: A statistical approach to defining meaningful change in psychotherapy research. J Consult Clin Psychol 59, 12-19.

[28] Sheng B, Cheng SK, Lau KK, Li HL, Chan, EL (2005) The effects of disease severity, use of corticosteroids and social factors on neuropsychiatric complaints in severe acute respiratory syndrome (SARS) patients at acute and convalescent phases. Eur Psychiatry 20, 236-242.

[29] Krendl AC, Perry BL (2021) The impact of sheltering in place during the COVID-19 pandemic on older adults' social and mental well-being. J Gerontol B Psychol Sci Soc Sci 76, e53-e58.

[30] De Pue S, Gillebert C, Dierckx E, Vanderhasselt MA, De Raedt R, Van den Bussche E (2021) The impact of the COVID-19 pandemic on wellbeing and cognitive functioning of older adults. Sci Rep 11, 4636.

[31] Chen LK (2020) Older adults and COVID-19 pandemic: Resilience matters. Arch Gerontol Geriatr 89, 104124.

[32] MacLeod S, Musich S, Hawkins K, Alsgaard K, Wicker ER (2016) The impact of resilience among older adults. Geriatr Nurs 37, 266-272.

[33] Ismail II, Kamel WA, Al-Hashel JY (2021) Association of COVID-19 pandemic and rate of cognitive decline in patients with dementia and mild cognitive impairment: A Cross-sectional study. Gerontol Geriatr Med 7, 23337214211005223.

[34] Newhouse P, Kellar K, Aisen P, White H, Wesnes K, Coderre E, Pfaff A, Wilkins H, Howard D, Levin ED (2012) Nicotine treatment of mild cognitive impairment: A 6-month doubleblind pilot clinical trial. Neurology 78, 91-101. 